\title{
Optimal Dispersal of Certificate Chains
}

\author{
Eunjin (EJ) Jung, Member, IEEE, Ehab S. Elmallah, Member, IEEE, and \\ Mohamed G. Gouda, Member, IEEE
}

\begin{abstract}
We consider a network where users can issue certificates that identify the public keys of other users in the network. The issued certificates in a network constitute a set of certificate chains between users. A user $u$ can obtain the public key of another user $v$ from a certificate chain from $u$ to $v$ in the network. For the certificate chain from $u$ to $v, u$ is called the source of the chain and $v$ is called the destination of the chain. Certificates in each chain are dispersed between the source and destination of the chain such that the following condition holds. If any user $u$ needs to securely send messages to any other user $v$ in the network, then $u$ can use the certificates stored in $u$ and $v$ to obtain the public key of $v$ (then $u$ can use the public key of $v$ to set up a shared key with $v$ to securely send messages to $v$ ). The cost of dispersing certificates in a set of chains among the source and destination users in a network is measured by the total number of certificates that need to be stored in all users. A dispersal of a set of certificate chains in a network is optimal if no other dispersal of the same chain set has a strictly lower cost. In this paper, we show that the problem of computing optimal dispersal of a given chain set is NP-complete. Thus, minimizing the total number of certificates stored in all users is NP-complete. We identify three special classes of chain sets that are of practical interests and devise three polynomial-time algorithms that compute optimal dispersals for each class. We also present two polynomial-time extensions of these algorithms for more general classes of chain sets.
\end{abstract}

Index Terms-Security and privacy protection, authentication, security and protection, authentication, certificate graph, certificate dispersal, public-key management.

\section{INTRODUCTION}

$\mathrm{W}_{\mathrm{n}}^{\mathrm{E}}$ E consider a network where users would like to send messages securely to other users. A user who would like to send a secure message is called a source and a user who is intended to receive such a message is called a destination.

In the Internet, it is common that one source may wish to send messages to many destinations. For example, a source Alice may wish to send her credit card number securely to several destination shopping sites, say, Amazon.com, eBay.com, and priceline.com. The secure communication between a source and a destination is protected by encrypting each exchanged message with a shared key known only to the source and destination.

In this network, each user $u$, whether source or destination, has a private key $r k_{u}$ and a public key $b k_{u}$. In order for a source $u$ to share a key $s k$ with a destination $v, u$ encrypts key $s k$ using the public key $b k_{v}$ of $v$ and sends the result, denoted $b k_{v}\{s k\}$, to $v$. Only $v$ can decrypt this message and obtain key $s k$ shared with $u$. This scenario necessitates that $u$ knows the public key $b k_{v}$ of $v$. In the above example, Alice needs to know the public keys of Amazon, eBay, and priceline.

- E. Jung is with the Department of Computer Science, The University of Iowa, 201L MacLean Hall, Iowa City, IA 52242.

E-mail: ejjung@cs.uiowa.edu.

- E.S. Elmallah is with the Department of Computing Science, University of Alberta, Edmonton, Alberta, Canada T6G 2H1.

E-mail: ehab@cs.ualberta.ca.

- M.G. Gouda is with the Department of Computer Sciences, University of Texas at Austin, 1 University Station (C0500), Austin, TX 78712-0233. E-mail: gouda@cs.utexas.edu.

Manuscript received 2 May 2005; revised 23 Feb. 2006; accepted 9 June 2006; published online 9 Jan. 2007.

Recommended for acceptance by X. Zhang.

For information on obtaining reprints of this article, please send e-mail to: tpds@computer.org, and reference IEEECS Log Number TPDS-0243-0505.

Digital Object Identifier no. 10.1109/TPDS.2007.1007.
If a user $u$ knows the public key $b k_{v}$ of another user $v$ in the network, then $u$ can issue a certificate, called a certificate from $u$ to $v$, that identifies the public key $b k_{v}$ of $v$. This certificate can be used by any user that knows the public key of $u$ to further acquire the public key of $v$.

A certificate from $u$ to $v$ is of the following form:

$$
r k_{u}\left\langle u, v, b k_{v}\right\rangle \text {. }
$$

This certificate is signed using the private key $r k_{u}$ of $u$, and it includes three items: the identity of the certificate issuer $u$, the identity of the certificate subject $v$, and the public key of the certificate subject $b k_{v}$. Any user that knows the public key $b k_{u}$ of $u$ can use $b k_{u}$ to obtain the public key $b k_{v}$ of $v$ from the certificate from $u$ to $v$. Note that when a user obtains the public key $b k_{v}$ of user $v$ from the certificate, the user not only finds out what $b k_{v}$ is, but also acquires the proof of the association that $b k_{v}$ is indeed the public key of user $v$.

The certificates issued by different users in a network can be represented by a directed graph, called the certificate graph of the network. Each node in the certificate graph represents a user in the network. Each directed edge from node $u$ to node $v$ in the certificate graph represents a certificate from $u$ to $v$ in the network.

Fig. 1 shows a certificate graph for a network with two sources, Alice and Bob, and six destinations, Amazon, eBay, priceline, Amex, Visa, and Discover. According to this graph,

Alice issues three certificates

(Alice, Amazon), (Alice, eBay),

and (Alice, priceline), and

Bob issues three certificates

(Bob, Amex), (Bob, Visa), and (Bob, Discover).

A more efficient way to support secure communication between the sources and the destinations is to introduce some intermediaries between the sources and the destinations. 


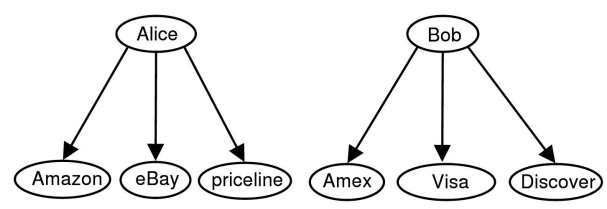

Fig. 1. A certificate graph of Alice and Bob.

The number of introduced intermediaries is much smaller than the number of sources and the number of destinations. Each intermediary has its own public and private key pair. The sources know the public keys of intermediaries and the intermediaries issue certificates of the public keys of the destinations. For example, two intermediaries, namely VeriSign and CertPlus, can be introduced between the two sources and the six destinations in Fig. 1. The result is the certificate graph in Fig. 2.

According to the certificate graph in Fig. 2, Alice needs to issue only one certificate to VeriSign and Bob needs to issue only one certificate to CertPlus. Alice can then use the two certificates (Alice,VeriSign) and (VeriSign,Amazon) to obtain the public key $b k_{A m a z o n}$, and so can securely send messages to Amazon. Also, Bob can use the two certificates (Bob,CertPlus) and (CertPlus, Visa) to obtain the public key $b k_{V i s a}$, and then can securely send messages to Visa.

Note that there is a certificate (VeriSign,Amex) in the certificate graph in Fig. 2 that is not needed to support secure communication between any source and any destination in Fig. 1. This redundancy is removed by specifying which "certificate chains" are being used by the sources and destinations. Certificate chains are defined as follows:

A simple path from a source $u$ to a destination $v$ in a certificate graph $G$ is called a chain from $u$ to $v$ in $G$. $u$ is the source of the chain and $v$ is the destination of the chain. For users $u$ and $v$ in a certificate graph $G$, if $u$ wishes to securely send messages to $v$, then there must be a chain from $u$ to $v$ in $G$. On the other hand, if there is a chain from $u$ to $v$, then $u$ does not necessarily wish to securely send messages to $v$. Fig. 3 shows the six chains that are needed to support the secure communications between the two sources and the six destinations in Fig. 1. Since Alice does not need to securely communicate with Amex, the certificate chain (Alice, VeriSign), (VeriSign, Amex) in the certificate graph in Fig. 2 is not included in Fig. 3.

The certificates in each chain need to be dispersed between the source and destination of the chain such that if a source $u$ wishes to securely send a message to a destination $v$, then $u$ can obtain the public key of $v$ from the set of certificates stored in $u$ and $v$. (Note that to "store a certificate in a user" does not necessarily mean that the user has a local copy of the certificate. Rather, it means that the



Fig. 2. A certificate graph with intermediaries.

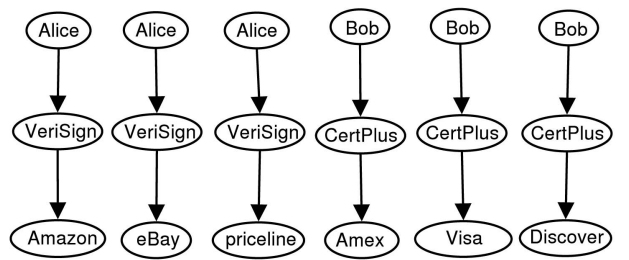

Fig. 3. Certificate chains from Fig. 2.

user only needs to know where to find the certificate, if a need for that certificate arises, either in its local storage or in a remote location.)

For example, assume that each source in Fig. 3 stores its certificate to the corresponding intermediary, and that each destination in Fig. 3 stores the certificate from its corresponding intermediary to itself. Thus,

Alice stores the certificate (Alice, VeriSign),

Bob stores the certificate (Bob, CertPlus),

Amazon stores the certificate (VeriSign, Amazon),

eBay stores the certificate (VeriSign, eBay),

priceline stores the certificate (VeriSign, priceline),

Amex stores the certificate (CertPlus, Amex),

Visa stores the certificate (CertPlus, Visa), and

Discover stores the certificate (CertPlus, Discover).

In this case, if Alice wishes to securely send messages to priceline, then Alice can use the two certificates stored in Alice's computer and the priceline Web site to obtain the public key of priceline and securely send the messages to priceline. Certificates that are not part of any chain are not stored because they are not needed. This is illustrated by the certificate (VeriSign, Amex), which appears in Fig. 2 but is not stored in Amex.

Dispersal of certificate chains and its cost are defined in Section 2. In Section 3, we show that finding an optimal dispersal of any set of chains is NP-complete. Thus, it becomes of interest to characterize the special cases of practical interest where the problem can be solved efficiently, as well as effective heuristic algorithms to solve general instances of problems. Subsequently, we identify three special classes of chain sets that are of practical interests and devise three polynomial-time algorithms that compute optimal dispersals for each class. For instance, the example dispersal above reflects the certificate dispersal in Secure Socket Layer (SSL). Such chain sets are defined as "short" chain sets in Section 4, and we present an algorithm that computes an optimal dispersal of any given short chain set. We also present two extensions of these algorithms for more general classes of chain sets.

\section{Certificate Dispersal}

In this section, we introduce definitions and notations to describe the optimal dispersal and prove two theorems of the properties of an optimal dispersal.

A certificate graph $G$ is a directed graph in which each directed edge, called a certificate, is a pair $(u, v)$, where $u$ and $v$ are distinct nodes in $G$. For each certificate $(u, v)$ in $G, u$ is called the issuer of the certificate and $v$ is called the subject of the certificate. Note that, according to this definition, no certificate has the same node as both its issuer and subject. 
A sequence of certificates $\left(v_{0}, v_{1}\right)\left(v_{1}, v_{2}\right) \cdots\left(v_{k-1}, v_{k}\right)$ in a certificate graph $G$, where the nodes $v_{0}, v_{1}, \cdots, v_{k}$ are all distinct, is called a chain from $v_{0}$ to $v_{k}$ in $G$. Node $v_{0}$ is called the source of the chain and node $v_{k}$ is called the destination of the chain. A set of chains in a certificate graph $G$ is called a chain set of $G$.

A dispersal $D$ of a chain set $C S$ assigns a set of certificates in $C S$ to each source node and each destination node in $C S$ such that the following condition holds. The certificates in each chain from a source node $u$ to a destination node $v$ in $C S$ are in the set D.u D.v, where D.u and D.v are the two sets of certificates assigned by dispersal $D$ to nodes $u$ and $v$, respectively. Thus, given a chain in $C S$, the source node $u$ and the destination node $v$ of the chain can find all the certificates in the chain in the set D.u D.v. When the source node $u$ and the destination node $v$ need to search for a chain from $u$ to $v$, then they can simply merge D.u and D.v to construct a certificate graph $G_{u, v}$, and search for a simple path from $u$ to $v$ in $G_{u, v}$. If there is a simple path from $u$ to $v$ in $G_{u, v}$, then this path is a certificate chain from $u$ to $v$. On the other hand, if there is no path from $u$ to $v$ in $G_{u, v}$, then nodes $u$ and $v$ recognize that there was no certificate chain in the given $C S$.

Let $D$ be a dispersal of a chain set $C S$. The cost of dispersal $D$, denoted cost.D, is the sum of the number of certificates in the sets assigned by dispersal $D$ to every source or destination node in $C S$ :

$$
\text { cost.D } \sum_{v \text { is a source or destination node in } C S}|D \cdot v| .
$$

A dispersal $D$ of a chain set $C S$ is optimal if and only if, for any other dispersal $D^{\prime}$ of the same chain set $C S$,

$$
\operatorname{cost} . D \leq \operatorname{cost} . D^{\prime} .
$$

In other words, an optimal dispersal $D$ of a chain set $C S$ minimizes the average number of certificates stored in each node.

Dispersal of a chain set is useful for many types of systems. We discuss three example types of systems here:

1. Deployed systems. In a deployed system, all the certificates are dispersed among the nodes in the system before the nodes start on a particular mission. For example, consider mobile units participating in a military operation. Chains that can be used for authentication are carefully chosen and dispersed. Each unit stores the assigned set of certificates by a dispersal of chosen chains. The units are deployed in mission, and when a unit needs to authenticate another unit, they do not have guarantee that any other unit will be available. Thanks to dispersal, these two nodes can use the certificates stored in each unit to find a certificate chain from one to the other. Many military applications fit in this type of systems.

2. Client-Server systems. In a client-server system, there are only a limited number of certificate authorities that issue certificates. In such systems, it is not necessary to collect all the certificates to optimally disperse them. For example, in Secure Socket Layer (SSL) systems, VeriSign is one of the few certificate authorities. A server, for example Amazon.com, does not need to know all the certificates in the system but only stores the certificate (Amazon.com, VeriSign). This is an optimal dispersal (more details are in Section 4) of this SSL system.

3. Evolving systems. In an evolving system, where certificates may be issued and revoked during the execution of the system, the system can start with an optimal dispersal of such system and gradually diverge from the dispersal. Even when the system diverges from its dispersal, it is still beneficial to start with an optimal dispersal as long as the changes in certificates are not a major portion of certificates in the system. Moreover, the dynamic dispersal protocol in [1] disperses newly issued certificates and revocation certificates so that the system stabilizes back to dispersal.

Let $(u, v)$ be a certificate that appears in one or more chains in a chain set $C S$, and let $D$ be a dispersal of $C S$. The location set of certificate $(u, v)$ assigned by $D$, denoted $D(u, v)$, is defined as a set of all nodes $x$ such that $(u, v)$ is in the set of certificates D.x. It is straightforward to show that the cost of dispersal $D$ equals $\sum_{(u, v) \in C S}|D(u, v)|$.

The location set $D(u, v)$ of a certificate $(u, v)$ assigned by a dispersal $D$ of a chain set $C S$ is optimal if and only if, for any other dispersal $D^{\prime}$ of $C S,|D(u, v)| \leq\left|D^{\prime}(u, v)\right|$.

Theorem 1. Let $D$ be a dispersal of a chain set CS. If $D$ is optimal, then, for every certificate $(u, v)$ in $C S$, the location set $D(u, v)$ is optimal.

Proof. The proof is by contradiction. Assume that $D$ is optimal, and there exists another dispersal $D^{\prime}$ of $C S$ where for some certificate $(u, v)$ in $C S,|D(u, v)|>\left|D^{\prime}(u, v)\right|$.

Now, consider the following assignment of certificates to each node in $C S$ :

$$
D^{\prime \prime}(x, y):= \begin{cases}D^{\prime}(x, y) & \text { if }(x, y)=(u, v), \\ D(x, y) & \text { if }(x, y) \neq(u, v) .\end{cases}
$$

Note that $D^{\prime \prime}$ is a dispersal of $C S$. This is true because, for any chain from a node $i$ to another node $j$ in $C S$, all the certificates in the chain are in $D^{\prime \prime} . i \cup D^{\prime \prime} . j$. Consider a certificate $(x, y)$ in the chain from $i$ to $j$ in $C S$, where $(x, y) \neq(u, v) . D(x, y)$ contains node $i$ or node $j$ by the definition of dispersal, so $D^{\prime \prime}(x, y)$ contains node $i$ or node $j$. In other words, any certificate $(x, y)$ in a chain from node $i$ to node $j$ in $C S$, where $(x, y) \neq(u, v)$, is in $D^{\prime \prime} . i \cup D^{\prime \prime} . j$. Similarly, for certificate $(u, v)$, if $(u, v)$ is in a chain from $i$ to $j$ in $C S, D^{\prime}(u, v)$ contains node $i$ or node $j$ by the definition of dispersal, so $D^{\prime \prime}(u, v)$ contains node $i$ or node $j$. In other words, if certificate $(u, v)$ is in a chain from node $i$ to $j$ in $C S$, then $(u, v)$ is in $D^{\prime \prime} . i \cup D^{\prime \prime} . j$. Therefore, for any given chain from a node $i$ to another node $j$ in $C S$, all the certificates in the chain are in $D^{\prime \prime} . i \cup D^{\prime \prime} . j$. Thus, $D^{\prime \prime}$ is a dispersal of $C S$.

The cost of dispersal $D^{\prime \prime}$ is computed as follows:

$$
\begin{aligned}
\text { cost. } D^{\prime \prime} & =\sum_{v \in C S}\left|D^{\prime \prime} . v\right| \\
& =\left(\sum_{\substack{(x, y) \in C S \\
(x, y) \neq(u, v)}}|D(x, y)|\right)+\left|D^{\prime}(u, v)\right| .
\end{aligned}
$$


By the assumption $\left|D^{\prime}(u, v)\right|<|D(u, v)|$,

$$
\begin{aligned}
\operatorname{cost} . D^{\prime \prime} & =\left(\sum_{\substack{(x, y) \in C S,(x, y) \neq(u, v)}}|D(x, y)|\right)+\left|D^{\prime}(u, v)\right| \\
& <\left(\sum_{\substack{(x, y) \in C S,(x, y) \neq(u, v)}}|D(x, y)|\right)+|D(u, v)| \\
& =\text { cost.D. }
\end{aligned}
$$

Thus, the cost of dispersal $D^{\prime \prime}$ is less than the cost of dispersal $D$ contradicting the assumption that $D$ is an optimal dispersal.

Therefore, the location set $D(u, v)$ assigned by an optimal dispersal $D$ is optimal for every certificate $(u, v)$ in $C S$.

Theorem 2. Let $D$ be a dispersal of a chain set CS. If, for every certificate $(u, v)$ in $C S$, the location set $D(u, v)$ is optimal, then $D$ is an optimal dispersal of CS.

Proof. The proof is by contradiction. Let $D$ be a dispersal for a chain set $C S$ and, for every certificate $(u, v)$ in $C S$, let the location set $D(u, v)$ be optimal. Also, let $D^{\prime}$ be another dispersal of $C S$, where cost. $D^{\prime}<\operatorname{cost} . D$. By the definition of the cost of dispersal,

$$
\begin{aligned}
\sum_{(u, v) \in C S}\left|D^{\prime}(u, v)\right| & =\text { cost. } D^{\prime}<\text { cost.D } \\
& =\sum_{(u, v) \in C S}|D(u, v)| .
\end{aligned}
$$

Thus, there must be at least one certificate $(u, v)$ in $C S$ such that $\left|D^{\prime}(u, v)\right|<|D(u, v)|$. This contradicts the definition of an optimal location set of $(u, v)$.

Therefore, if $D(u, v)$ is optimal for every certificate $(u, v)$ in a chain set $C S$, then $D$ is an optimal dispersal of $C S$.

\section{NP-Completeness of Optimal Dispersal of Chain Sets}

In this section, we show that the chain dispersal problem is NP-complete by a reduction from the vertex cover problem. For convenience, these two problems are described below:

- The Vertex Cover (VC) Problem. Given a connected graph $G$ and a positive integer $k$, we ask if there exists a vertex cover of size $\leq k$. Any instance of this problem can be represented by the pair $(G, k)$. For directed graphs, the VC problem can be defined similarly by ignoring the directions associated with the arcs; the resulting problem on directed graphs remains NP-complete.

- The Certificate Dispersal (CD) Problem. Given a chain set $C S$ and a positive integer $m$, we ask if there exists a dispersal $D$ of $C S$ such that cost. $D \leq m$. Any instance of this problem can be represented by the pair $(C S, m)$.

\section{Theorem 3. $C D$ is NP-complete.}

Proof. First, we show that $C D$ is in NP. Given an instance $(C S, m)$ of $C D$, and a dispersal $D$ of $C S$ with cost.D $\leq m$, one can verify in polynomial-time that $D$ is indeed a dispersal of $C S$ and cost. $D \leq m$. To verify that $D$ is a dispersal of $C S$, one checks that all the certificates in each chain from a node $u$ to another node $v$ in $C S$ are in $D . u \cup D . v$. Once $D$ is verified as a dispersal, cost.D is computed as the sum of $|D . u|$ for each node $u$ in $C S$ and can be compared to $m$. The time complexity of this verification step is $O(p \times n)$, where $p$ is the number of chains in the chain set and $n$ is the length of the longest chain in $C S$.

Second, we show that $\mathrm{VC}$ reduces to $\mathrm{CD}$ in polynomialtime. Given an instance $(G, k)$ of $\mathrm{VC}$, we construct an instance $(C S, m)$ of $C D$ such that the $C D$ instance has a yes answer if and only if the given VC has a yes answer. The construction is as follows:

1. For each edge $(u, v)$ in $G, C S$ has a chain

$$
(u, x)(x, y)(y, v)
$$

of length 3 .

2. Let $n^{+}$be the number of nodes that have outgoing edges in $G$, and let $n^{-}$be the number of nodes that have incoming edges in $G$. Set $m=n^{+}+n^{-}+k$.

$(\mathbf{C D} \Leftarrow \mathbf{V C})$. We now show that if the instance $(G, k)$ of VC has a yes answer, then the corresponding instance $(C S, m)$ of $C D$ has a yes answer. Let $X$ be a vertex cover of $G$, where $|X| \leq k$. For each node $u$ in the cover $X$, assign certificate $(x, y)$ in $C S$ to $D . u$. For each node $u$ in $G$, if there exists $(u, x)$ in $C S$, then assign certificate $(u, x)$ to $D$.u. For each node $v$ in $G$, if there exists $(y, v)$ in $C S$, then assign certificate $(y, v)$ to D.v. In the following two steps, we prove that $D$ is a dispersal of $C S$ whose cost is at most $m$ :

1. $D$ is a dispersal of $C S$. For any chain in $C S$ from a node $u$ to a node $v$, the chain consists of three certificates $(u, x),(x, y)$, and $(y, v)$. Certificate $(u, x)$ is stored in D.u and certificate $(y, v)$ is stored in D.v. For certificate $(x, y),(x, y)$ is stored in every node in the vertex cover of $G$. By the definition of the vertex cover, for each edge $(u, v)$ in $G$, the vertex cover contains node $u$ or node $v$. Certificate $(x, y)$ is assigned to every node in the vertex cover of $G$, so $(x, y)$ is stored in D.u or D.v. Thus, every certificate in the chain from $u$ to $v$ is stored in $D . u \cup D . v$, as required by the definition of dispersal.

2. cost. $D \leq m$. For each node $u$ in $G$ that has any outgoing edges, there is certificate $(u, x)$ in $C S$ that is assigned only to node $u$ by $D$. Similarly, for each node $v$ in $G$ that has any incoming edges, there is certificate $(y, v)$ in $C S$ that is assigned only to node $v$ by $D$. For certificate $(x, y),(x, y)$ is assigned to all the nodes in the vertex cover, so $(x, y)$ is assigned to at most $k$ nodes. In total, cost. $D$ is at most $m=\left(k+n^{+}+n^{-}\right)$.

The above argument shows that $D$ is a dispersal of constructed $C S$ and cost. $D \leq m$. This proves that if an instance of VC $(G, k)$ has a yes answer, then the corresponding instance of $\mathrm{CD}(C S, m)$ has a yes answer.

$(\mathbf{C D} \Rightarrow \mathbf{V C})$. We now show that if the constructed instance $(C S, m)$ of $C D$ has a yes answer, then the given instance $(G, k)$ of VC has a yes answer. Let $D$ be a 
dispersal of $C S$, where cost. $D \leq m$. For every edge $(u, v)$ in $G$, there is chain $(u, x)(x, y)(y, v)$ in $C S$. For certificates $(u, x)$ and $(y, v)$, they will be assigned to at least one node, so $|D(u, x)| \geq 1$ and $|D(y, v)| \geq 1$. The number of such $(u, x)$ certificates is $n^{+}$and the number of such $(y, v)$ certificates is $n^{-}$. So, certificate $(x, y)$ is assigned to at most $k$ nodes, where $k$ is $m-n^{+}-n^{-}$. In other words, $|D(x, y)| \leq k$.

Now, for each edge $(u, v)$ in $G$, there is chain $(u, x)$ $(x, y)(y, v)$ in $C S$, and $(x, y)$ is stored in D.u $\cup$ D.v. In other words, for each edge $(u, v)$ in $G$, the location set of $D(x, y)$ contains node $u$ or node $v$. Therefore, the location set of $D(x, y)$ is a vertex cover of $G$. The size of the location set $D(x, y)$ is at most $k$, so the size of the vertex cover is at most $k$, and the instance $(G, k)$ of $\mathrm{VC}$ has a yes answer.

In conclusion, the above proof shows that CD is in NP and $\mathrm{VC}$ reduces to $\mathrm{CD}$ in polynomial-time. Therefore, $\mathrm{CD}$ is NP-complete.

In light of the above complexity result, it becomes of importance to identify special classes of chain sets of practical interest for which the problem can be solved efficiently. This direction is pursued in the following cases:

1. Short chain sets. In Section 4, we start by investigating the class of chain sets, where each chain is of length at most 2. This class of chain sets is the one currently being used in the Secure Socket Layer (SSL) protocol. Recall that the chain set in the example in Fig. 3 in Section 1 falls into this class.

2. Disconnected chain sets. In Section 5, we investigate the class of chain sets where, for a given certificate, no node can be both the source and the destination of any chain that contains this certificate. This reflects a system where the authentication is needed in an asymmetric manner. For example, when there are clients and servers in the system, one can imagine that clients would use certificates to authenticate servers, while servers would use passwords to authenticate clients. Such asymmetric systems can be represented as this class of chain sets.

3. Concise graphs. In Section 6, we investigate the class of chain sets where the chains are derived from acyclic certificate graphs. This class reflects systems where the need for authentication is unidirectional. For example, any hierarchical system where a lower level user is authenticated by a higher level user, but not the other way around, would be represented by an acyclic certificate graph.

For all these three classes of chain sets, we present polynomial-time algorithms that compute optimal dispersals of chain sets in each class and prove their optimality.

Also below, we identify two classes of parameterized chain sets that are defined using an integer parameter $k$. In the first class, each chain set has at most $k$ chains with three or more certificates. In the second class, each chain set has at most $k$ nodes that may act both as sources and destinations. For both classes, we obtain polynomial-time algorithms that compute optimal dispersals when $k$ is fixed.

\section{Optimal Dispersal of Short Chain Sets}

In the previous section, we proved that computing an optimal dispersal of any chain set, which includes chains whose length is 3 or more, is NP-complete. In this section,

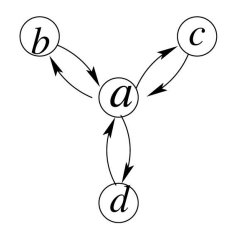

(a)

$$
\begin{array}{rr}
(b, a)(a, c), & (d, a)(a, b), \\
(c, a)(a, b), & (c, a)(a, d), \\
(b, a)(a, d), & (d, a)(a, c)\}
\end{array}
$$

(b)
Fig. 4. An example of a short chain set.

we show that there is a polynomial-time algorithm that computes an optimal dispersal of any chain set whose chains are all of length at most 2 . This class of chain sets is currently in use in the Internet in Secure Socket Layer (SSL).

A chain set $C S$ is short if and only if the length of the longest chain in $C S$ is at most 2. For example, consider the star certificate graph in Fig. 4a. In this certificate graph, assume that each satellite node, $b, c$, or $d$, wishes to securely communicate with every other satellite node. Fig. 4 b shows the resulting short chain set.

Algorithm 1 computes an optimal dispersal of a short chain set. Consider the certificate $(b, a)$ in the example short chain set in Fig. 4. Chains that have $(b, a)$ are $(b, a)(a, c)$ and $(b, a)(a, d)$. So, $b$ is the source of every chain that has $(b, a)$. Therefore, Algorithm 1 assigns $(b, a)$ to D.b. After considering all the certificates in the short chain set, the optimal dispersal is computed by Algorithm 1 as follows:

$$
\begin{aligned}
\{D \cdot a & =\{\}, D . b=\{(a, b),(b, a)\}, \\
D . c & =\{(a, c),(c, a)\}, D . d=\{(a, d),(d, a)\}\} .
\end{aligned}
$$

ALGORITHM 1: optimal dispersal of short chain sets

INPUT: a short chain set $C S$

OUTPUT: a dispersal $D$ of $C S$

STEPS:

1: for each node $u$ in $C S, D . u:=\{\}$

2: for each certificate $(u, v)$ in $C S$ do

3: $\quad$ if there is a node $x$ such that

the source or destination

of every chain that has $(u, v)$ is $x$

then add $(u, v)$ to $D . x$

else add $(u, v)$ to both D.u and D.v

Theorem 4. Given a short chain set CS, the dispersal D of CS computed by Algorithm 1 is optimal.

Proof. The proof consists of two parts. First, we show that Algorithm 1 computes a dispersal $D$. Second, we show that $D$ is optimal.

Proof of the First Part. By the definition of dispersal in Section 2, if all the certificates in each chain from a source node $u$ to a destination node $v$ in $C S$ are in set D.u $\cup$ D.v, then $D$ is a dispersal of $C S$. In other words, if a certificate $(u, v)$ is stored in the source or destination nodes of every chain that contains $(u, v)$, then $D$ is a dispersal.

By Algorithm 1, every certificate $(u, v)$ is stored either in D.x of some node $x$, or both D.u and D.v. Since the maximum length of a chain in $C S$ is 2, every chain that contains $(u, v)$ starts at $u$ or ends at $v$. Hence, if $(u, v)$ is 


$$
\begin{aligned}
& \{(d, a), \\
& (a, b)(b, c), \\
& (a, c)(c, d), \\
& (a, b)(b, c)(c, d)(d, e)\}
\end{aligned}
$$

Fig. 5. An example of disconnected chain set.

stored in both D.u and D.v, then certificate $(u, v)$ is stored in the source or destination node of every chain that contains $(u, v)$. If $(u, v)$ is stored in node $x$, then, by Algorithm 1, $x$ is either the source node or the destination node of every chain that contains $(u, v)$. Therefore, $(u, v)$ is stored in the source or the destination node of every chain that contains $(u, v)$.

Proof of the Second Part. The proof is by contradiction. Let $D$ be the dispersal of a short chain set $C S$ computed by Algorithm 1 and let $D^{\prime}$ be another dispersal of $C S$. Assume that cost. $D^{\prime}<$ cost.D. There must be at least one certificate $(u, v)$ such that $\left|D^{\prime}(u, v)\right|<|D(u, v)|$.

Let $(u, v)$ be such a certificate, $\left|D^{\prime}(u, v)\right|<|D(u, v)|$. By Algorithm 1, $|D(u, v)|$ is either 1 (if there exists some node $x$ that is the source or destination node of every chain that has $(u, v))$ or 2 (otherwise). Therefore, $\left|D^{\prime}(u, v)\right|=1$ and $|D(u, v)|=2$, and there exists no node $x$ in $C S$ that is the source or destination node of every chain that has $(u, v)$. By the definition of dispersal, the node $w$ in $D^{\prime}(u, v)$ should be the source or a destination of every chain that contains $(u, v)$ in $C S$. This contradicts that there exists no node $x$ in $C S$ such that $x$ is the source or destination node of every chain that has $(u, v)$.

Therefore, cost. $D \leq$ cost. $D^{\prime}$ for any dispersal $D^{\prime}$ of $C S$. Algorithm 1 computes an optimal dispersal of a short chain set $C S$.

The time complexity of Algorithm 1 is $O(e p)$, where $e$ is the number of certificates in the input short chain set and $p$ is the number of chains in the chain set.

\section{Optimal Dispersal of Disconnected Chain Sets}

In this section, we identify a special class of chain sets and present an algorithm that computes an optimal dispersal for this class of chain sets in polynomial-time. A chain set $C S$ is disconnected if and only if for every certificate $(u, v)$ in $C S$, the set of source nodes of the chains that contain $(u, v)$ and the set of destination nodes of the chains that contain $(u, v)$ are disjoint. This reflects a system where the authentication is performed in an asymmetric manner. For example, when there are clients and servers in the system, one can imagine that clients would use certificates to authenticate servers, while servers would use passwords to authenticate clients. Such asymmetric systems can be represented as disconnected chain sets. Fig. 5 shows an example of a disconnected chain set.

$(d, a)$ has the set of source nodes $\{d\}$ and the set of destination nodes $\{a\}$, which are disjoint. $(a, b)$ has the set of source nodes $\{a\}$ and the set of destination nodes $\{c, e\}$, which are disjoint. Every certificate in this chain set has disjoint sets of source and destination nodes.
Algorithm 2 computes an optimal dispersal of a disconnected chain set. Consider certificate $(a, b)$ in the example disconnected chain set in Fig. 5. Algorithm 2 constructs a bipartite graph $G^{\prime}$ for certificate $(a, b)$, where $G^{\prime}=\left(V^{\prime}, E^{\prime}\right), \quad V^{\prime}=\{a, c, e\}$, and $E^{\prime}=\{(a, c),(a, e)\}$. The vertex cover of minimum size of $G^{\prime}$ is $\{a\}$. Thus, $(a, b)$ is stored in D.a. After considering all certificates in the chain set, the example disconnected chain set is optimally dispersed by Algorithm 2 as follows:

$$
\begin{aligned}
& \{D \cdot a=\{(a, b),(b, c),(c, d)\}, D \cdot b=\{\}, D \cdot c=\{\}, \\
& \text { D.d }=\{(a, c),(d, a)\}, D \cdot e=\{(d, e)\}\} .
\end{aligned}
$$

ALGORITHM 2: optimal dispersal of disconnected chain sets

INPUT: a disconnected chain set $C S$

OUTPUT: a dispersal $D$ of $C S$

STEPS:

1: for each node $u$ in $G, D \cdot u:=\{\}$

2: for each certificate $(u, v)$ in $G$ do

3: $\quad G^{\prime}=\left(V^{\prime}, E^{\prime}\right)$ where $V^{\prime}=\{\}$ and $E^{\prime}=\{\}$

4: $\quad$ for each chain from node $x$ to node $y$ that contains $(u, v)$ do

5: $\quad$ add nodes $x$ and $y$ to $V^{\prime}$

6: $\quad$ add $(x, y)$ to $E^{\prime}$

7: $\quad$ compute a minimal vertex cover of the bipartite graph $G^{\prime}$

8: $\quad$ add $(u, v)$ to each node in the vertex cover

Theorem 5. Given a disconnected chain set CS, the dispersal D of CS computed by Algorithm 2 is optimal.

Proof. The proof consists of two parts. First, we show that Algorithm 2 produces a dispersal. Second, we show that the resulting dispersal is optimal.

Proof of the First Part. Let D.u be the set of certificates assigned to a node $u$ in $C S$ by Algorithm 2. Consider any certificate $(u, v)$ in a chain from a source node $x$ to a destination node $y$ in $C S$. By Algorithm 2, since there is a chain from $x$ to $y$ that goes through $(u, v)$, there is an edge $(x, y)$ in $G^{\prime}$ for $(u, v)$. By the definition of vertex cover, for edge $(x, y)$ in $G^{\prime}$, node $x$ or node $y$ is in the vertex cover. Therefore, for the chain from $x$ to $y,(u, v)$ is stored in D.x or D.y. This is true for all the certificates in the chain from $x$ to $y$, for any chain in $C S$. Hence, $D$ satisfies the dispersal condition in Section 2, so $D$ is a dispersal of $C S$.

Proof of the Second Part. By Theorem 2, if we can find a dispersal $D$ where $D(u, v)$ of every certificate $(u, v)$ in $C S$ is optimal, then $D$ is an optimal dispersal of $C S$. So, we only need to prove that a dispersal computed by Algorithm 2 produces an optimal location set of each certificate in $C S$. The proof is by contradiction. Assume there is another dispersal $D^{\prime}$ of $C S$, where cost. $D^{\prime}<$ cost.D. There must be at least one certificate $(u, v)$ where $\left|D^{\prime}(u, v)\right|<|D(u, v)|$. For every chain from a node $x$ to a node $y$ that contains $(u, v), D^{\prime}(u, v)$ should contain $x$ or $y$. Therefore, $D^{\prime}(u, v)$ is a vertex cover of the bipartite graph $G^{\prime}$ constructed for $(u, v)$, where

$$
\left|D^{\prime}(u, v)\right|<|D(u, v)|
$$




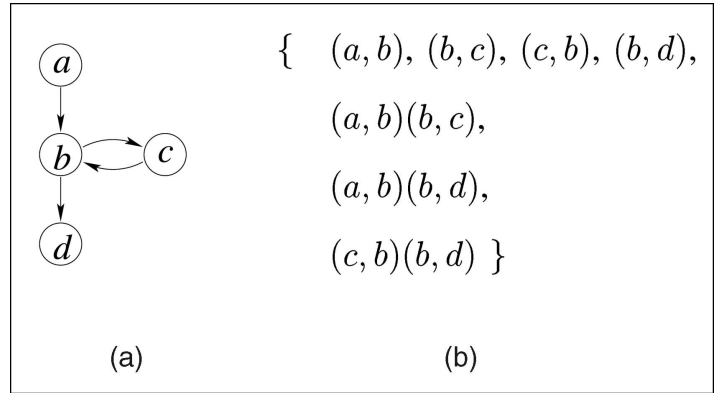

Fig. 6. An example of a concise certificate graph and a derivable chain set.

This contradicts that $D(u, v)$ is the vertex cover of minimum size of $G^{\prime}$ by line 7 in Algorithm 2. Therefore, $D(u, v)$ is an optimal location set of $(u, v)$ for every certificate $(u, v)$ in $C S$. By Theorem 2, D is optimal.

For each certificate $(u, v)$, the graph $G^{\prime}$ constructed for $(u, v)$ is a bipartite graph. It is because the set of source nodes of the chains that contain $(u, v)$ and the set of the destination nodes of the chains that contain $(u, v)$ are disjoint by the definition of disconnected chain set. Finding a vertex cover in a bipartite graph is a well-known problem in graph theory, which takes $O\left(n^{\prime} e^{\prime}\right)$ steps, where $n^{\prime}$ is the number on nodes in $G^{\prime}$ and $e^{\prime}$ is the number of edges in $G^{\prime}$. In the worst case, $n^{\prime}=n$ and $e^{\prime}=p$, where $n$ is the number of nodes in $C S$, and $p$ is the number of chains in $C S$. Therefore, the time complexity of Algorithm 2 is $O(e \times n p)=O(e n p)$, where $e$ is the number of certificates in $C S$.

\section{Optimal Dispersal of Concise Graphs}

In this section, we present an algorithm that computes optimal dispersal for chain sets "derivable" from a class of certificate graphs called concise certificate graphs. A certificate graph $G$ is called concise if and only if it satisfies the following two conditions:

1. Short Cycles. Every simple directed cycle in $G$ is of length 2.

2. Nonredundancy. $G$ has at most one chain from any node to any other node.

Concise certificate graphs represent many useful certificate systems. For example, a hierarchical certificate system would typically generate a tree-shaped certificate graph. Any tree-shaped certificate graph is a concise certificate graph.

Fig. 6a shows an example of a concise certificate graph. Note that, in a concise graph, there can be two opposite direction certificates between two adjacent nodes. We refer to any such pair of certificates as twins, and we refer to each one of those certificates as the twin certificate of the other. In the concise graph in Fig. 6a, the two certificates $(b, c)$ and $(c, b)$ are twins.

A chain set is derivable from some certificate graph $G$ if and only if the chain set consists of all the certificate chains in $G$. For example, the chain set in Fig. $6 \mathrm{~b}$ is derivable from the certificate graph in Fig. 6a.

Algorithm 3 computes an optimal dispersal of a concise certificate graph. Consider certificate $(b, c)$ in the example concise certificate graph in Fig. 6a. Algorithm 3 computes the set of nodes from which there is a chain to $b$, denoted $R . b$, as $\{a, b\}$. Also, Algorithm 3 computes the set of nodes to which there is a chain from $c$, denoted R.c as $\{c\}$. $|R . b|>|R . c|$, so $(b, c)$ is stored in $c$. After considering all the certificates in the graph, the example concise certificate graph is optimally dispersed by Algorithm 3 as follows:

$$
\begin{aligned}
\text { D. } a & =\{(a, b)\}, D \cdot b=\{(c, b)\}, \\
D \cdot c & =\{(b, c)\}, D \cdot d=\{(b, d)\} \quad\} .
\end{aligned}
$$

ALGORITHM 3: optimal dispersal of concise certificate graphs

INPUT: a concise certificate graph $G$

OUTPUT: a dispersal $D$ of the chain set $C S$

derivable from $G$

STEPS:

1: for each node $u$ in $G, D . u:=\{\}$

2: for each certificate $(u, v)$ in $G$ do

3: compute the set R.u that contains $u$ and every node $x$ from which there is a chain to $u$ in $G$ and this chain does not contain the twin certificate $(v, u)$

4: $\quad$ compute the set R.v that contains $v$ and every node $x$ to which there is a chain from $v$ in $G$ and this chain does not contain the twin certificate $(v, u)$

5: $\quad$ if $|R . u| \leq|R . v|$

6: $\quad$ then for every node $x$ in R.u, add $(u, v)$ to D.x

7: $\quad$ else for every node $y$ in $R . v$, add $(u, v)$ to D.y

Theorem 6. Given a concise certificate graph $G$, the dispersal $D$ of the chain set $C S$ derivable from $G$ computed by Algorithm 3 is optimal.

The proof is in [2]. The complexity of Algorithm 3 is $O(e n)$, where $e$ is the number of certificates in the input concise certificate graph and $n$ is the number of nodes in the concise certificate graph.

\section{Optimal Dispersal of $k$-Long Chain Sets}

In Section 3, we showed that computing an optimal dispersal of any chain set, which includes chains of length 3 or more, is $\mathrm{NP}$-complete. If all the chains in a chain set are of length at most 2, i.e., if the chain set is short, then we can use Algorithm 1 in Section 4 to compute an optimal dispersal of the short chain set. In this section, we consider a more general class of chain sets where, there are a fixed number $k, k \geq 1$, of chains of length greater than 2. Consideration of such chain sets is motivated, for instance, by the following example: Consider a hierarchical network made of a number of autonomous systems. Certificate chains within any single autonomous system are expected to be short, whereas certificate chains that span multiple autonomous systems are expected to be long. The chain set of these autonomous systems contain mostly short intra-chains, but may contain a fixed number of long inter-chains. Our main result here is a polynomial-time algorithm that computes an optimal dispersal for such chain set for fixed $k$.

In this section, we present Algorithm 4, which computes an optimal dispersal of a chain set where there are $k$ chains of length greater than 2 for some constant $k$. We call such sets k-long chain sets. Roughly speaking, our general 




Fig. 7. An example of a 1-long chain set.

strategy is to consider all possible ways of assigning certificates that appear in long chains to the relevant source and destination nodes, and then handling the remaining short chains with the aid of Algorithm 1. To develop some initial intuition, first we show how to compute an optimal dispersal of an example 1-long chain set in Fig. 7b, and then we show how to generalize for $k$-long chain sets.

Let $C S$ be the 1-long chain set in Fig. $7 \mathrm{~b}$, which is a chain set of the certificate graph in Fig. 7a. There is one long chain $(c, a)(a, b)(b, d)$ and three other short chains. There are three types of certificates in this chain set:

1. Certificates used only in long chains: for example, $(b, d)$.

A certificate of this type can be dispersed either to the source or to the destination of each long chain that contains this certificate. For example, certificate $(b, d)$ in $C S$ is used only in the long chain and needs to be dispersed either to $c$ or to $d$. This certificate is not used in any other chains, so it does not change the cost of dispersal whether it is dispersed to $c$ or $d$.

2. Certificates used only in short chains: for example, $(b, c)$.

For certificates of the second type, we can use Algorithm 1 in Section 4 to disperse such certificates. For example, certificate $(b, c)$ is dispersed to node $a$ by Algorithm 1.

3. Certificates used in both long and short chains: for example, $(a, b),(c, a)$.

Dispersing a certificate of the third type needs to consider every possible assignment of this certificate among sources and destinations of long chains. For example, certificate $(a, b)$ is used in three chains, $(a, b)(b, c), \quad(c, a)(a, b)$, and $(c, a)(a, b)(b, d)$. If we choose to disperse $(a, b)$ to the source $c$ of long chain, then we do not need to disperse $(a, b)$ to any other node in $C S$, since $c$ happens to be source or destination of all the short chains that contain $(a, b)$. By contrast, if we choose to disperse $(a, b)$ to the destination $d$ of long chain, then we need to disperse $(a, b)$ to other nodes than $d$ since $d$ is neither source nor destination of two short chains $(a, b)(b, c)$ and $(c, a)(a, b)$. In other words, $D(a, b)$ could be either $\{c\}$ or $\{a, b, d\}$, depending on whether $(a, b)$ is assigned to the source or the destination of the long chain. This shows that for each certificate of the third type that is used in both long and short chains, in each assignment of this certificate in sources and destinations of long chains, we need to check which short chains still needs dispersal of this certificate.
After considering all three types of certificates in $C S$, the resulting optimal dispersal of $C S$ in Fig. $7 \mathrm{~b}$ becomes as follows:

$$
\begin{aligned}
\{\quad D \cdot a & =\{(b, c)\}, D \cdot b=\{(c, a)\}, \\
D \cdot c & =\{(c, a),(a, b)\}, D \cdot d=\{(b, d)\} \quad\} .
\end{aligned}
$$

To extend this solution for 1 -long chain set to $k$-long chain sets, we need to define a terminal set of a chain set. A terminal set of a chain set $C S$ is a subset of nodes in $C S$ that consists of the source or destination of each chain in $C S$. For example, the four nodes $a, b, c$, and $c$ are the sources of all four chains in the chain set in Fig. $7 \mathrm{~b}$, so $\{a, b, c\}$ is a terminal set of this chain set. Algorithm 4 computes an optimal dispersal of $k$-long chain sets using this terminal set.

ALGORITHM 4: optimal dispersal of $k$-long chain sets

INPUT: a $k$-long chain set $C S$

OUTPUT: a dispersal $D$ of $C S$ STEPS:

1: for each node $u$ in $C S, D . u:=\{\}$

2: for each certificate $(u, v)$ in $C S$ do

3: $\quad$ compute the chain set $L S$ of all long chains that contain $(u, v)$ in $C S$

4: $\quad$ for each possible terminal set $X$ of $L S$

5: $\quad$ for each node $w$ in $C S$, if $w \in X$ then

$D_{X} \cdot w:=\{(u, v)\}$ else $D_{X} \cdot w:=\{\}$

6: $\quad$ compute the chain set $S$ of all the chains that contain $(u, v)$ and their sources and destinations are not in $X$

7: $\quad$ run Algorithm 1 on $S$ and add the resulting location set of $(u, v)$ to $D_{X}$

8: $\quad$ find $D_{X}$ with the minimal cost

9: $\quad$ for each node $u$ in $C S$, add $D_{X} . u$ to D.u

Consider $(c, a)$ in the example chain set in Fig. $7 \mathrm{~b}$. The set of all long chains that contain $(c, a)$, denoted $L S$ in Algorithm 4 , is $\{(c, a)(a, b)(b, d)\}$. For a terminal set $\{c\}$, $(c, a)$ is dispersed to node $c$ and the set of remaining short chains, denoted $S$ in Algorithm 4, becomes $\{(b, c)(c, a)\}$. There is node $b$ that is the source of every chain in $S$, so $(c, a)$ is dispersed to node $b$. The resulting dispersal of $(c, a)$, $\{b, c\}$, is an optimal location set of $(c, a)$. After considering every certificate, the dispersal of the example chain set in Fig. $7 \mathrm{~b}$ computed by Algorithm 4 becomes the same with the dispersal above, and this dispersal is optimal. Theorem 7 shows that Algorithm 4 computes an optimal dispersal of a given $k$-long chain sets.

Theorem 7. Given a k-long chain set CS, the dispersal D of the chain set CS computed by Algorithm 4 is optimal.

The proof is in [2]. The time complexity of this algorithm is $O\left(2^{k} \times e p\right)$, where $k$ is the number of long chains in $C S, e$ is the number of certificates in $C S$, and $p$ is the number of chains in $C S$. This complexity is computed as follows: The number of terminal sets for $k$ long chains is $O\left(2^{k}\right)$, and, for each terminal set, the number of short chains to consider is $O(p)$. We repeat this procedure for $e$ certificates. Since $k$ is a constant, the time complexity becomes $O(e p)$. 


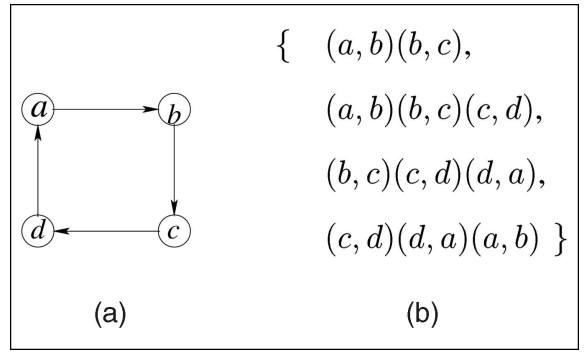

Fig. 8. An example of a 1-connected chain set.

\section{Optimal Dispersal of $k$-Connected Chain Sets}

In Section 5, we presented Algorithm 2, which computes an optimal dispersal of a disconnected chain set. In this section, we investigate a more general class of chain sets, where there are at most $k$ nodes in the intersection of the source set and the destination set of each certificate in a chain set. We call such chain sets $k$-connected chain sets. This class of chain sets models a client-server system that uses two different authentication methods. As discussed in Section 5, in some client-server systems, clients authenticate servers via certificates, whereas servers authenticate clients via other means, e.g., passwords. However, there may be a few mutual authentications via certificates between servers. These certificates used by servers may have nonempty intersections of the source and destination sets. Such clientserver systems can be represented as $k$-connected chain sets.

Fig. $8 \mathrm{~b}$ shows an example of a 1-connected chain set, which is a chain set of the certificate graph in Fig. 8a. For certificate $(a, b)$, the sources of the chains that contain $(a, b)$ are $\{a, c\}$ and the destinations of such chains are $\{b, c, d\}$. The intersection of two sets is $\{c\}$. Similarly, the cardinality of the intersection set is at most 1 for every certificate in this chain set, so the chain set in Fig. $8 \mathrm{~b}$ is 1-connected.

Assume that $(a, b)$ is stored in D.c in some dispersal $D$ of this chain set. The remaining chain to be dispersed is $(a, b)(b, c)(c, d)$. Certificate $(a, b)$ can be stored either in D.a or in D.d, either of which makes no difference in the dispersal cost. Or assume that $(a, b)$ is not stored in $D^{\prime} . c$ in some dispersal $D^{\prime}$ of this chain set. Certificate $(a, b)$ needs to be stored in $D^{\prime}$.a and $D^{\prime} . b$. We can repeat this process for each certificate to find the dispersal as follows:

$$
\left.\begin{array}{rlrl}
\{D \cdot a & =\{(a, b),(b, c)\}, & D \cdot b & =\{(c, d),(d, a)\}, \\
D \cdot c & =\{(a, b)\}, & & D \cdot d=\{(c, d)\}
\end{array}\right\} .
$$

This is also an optimal dispersal of this 1-connected chain set.

To extend this solution for 1-connected chain sets to $k$-connected chain sets, we need to define an intersection set of a certificate. An intersection set of a certificate $(u, v)$ in a chain set $C S$ is a set of nodes that appear both in the set of sources and the set of destinations of the chains that contain $(u, v)$. For certificate $(a, b)$ in Fig. $8 \mathrm{~b}$, the sources of the chains that contain $(a, b)$ are $\{a, c\}$ and the destinations of such chains are $\{b, c, d\}$. The intersection of two sets is $\{c\}$, so $\{c\}$ is the intersection set of $(a, b)$. Algorithm 5 computes an optimal dispersal of $k$-connected chain sets using this intersection set.
ALGORITHM 5: optimal dispersal of $k$-connected chain sets

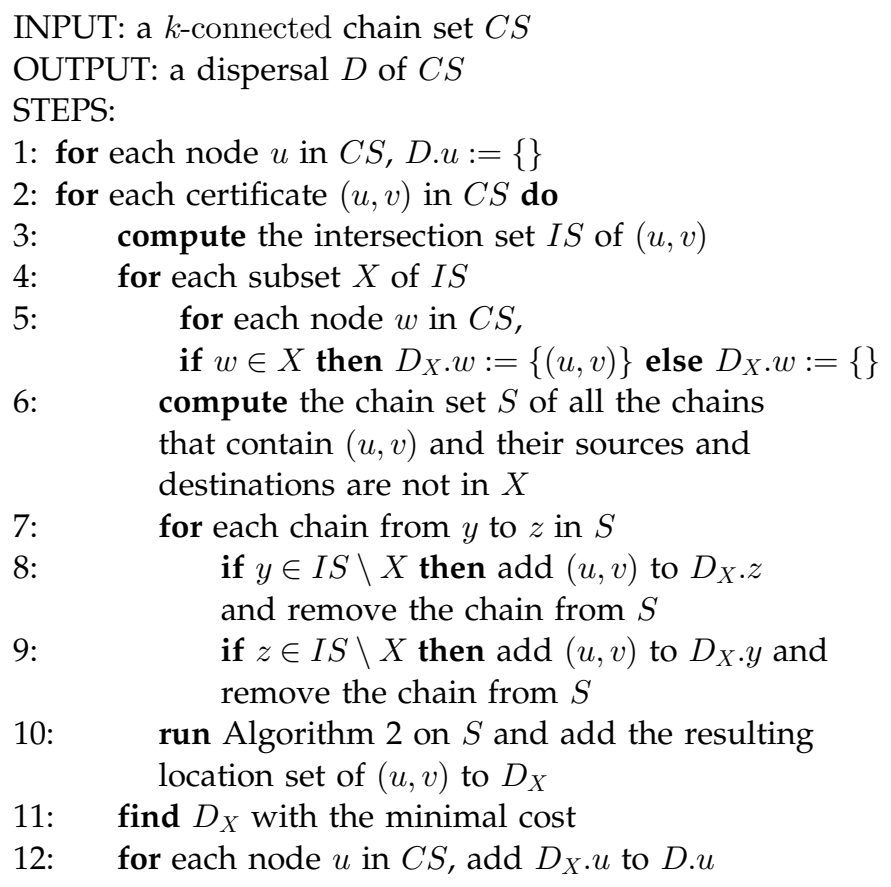

The proof of the optimality of this algorithm is straightforward. Since this algorithm considers every possible subset of the intersection set, it is guaranteed to find the optimal location set of each certificate. By Theorem 2, the dispersal computed by this algorithm is optimal.

The time complexity of this algorithm is $O\left(2^{k} \times e n p\right)$, where $k$ is the tight upper bound of the number of nodes in intersection sets of all the certificates in $C S, n$ is the number of nodes in $C S, e$ is the number of certificates in $C S$, and $p$ is the number of chains in $C S$. Since there are at most $k$ nodes in the intersection set of each certificate, there are at most $2^{k}$ subsets of the intersection set. For each subset, we run Algorithm 2, whose complexity is $O(e n p)$. Therefore, the total time complexity becomes $O\left(2^{k} e n p\right)$. Since $k$ is a constant, the time complexity becomes $O(e n p)$.

\section{Related Work}

Several papers have investigated the use of certificates for confidentiality, authentication, and authorization. We summarize the results of these papers in the following paragraphs:

Architectures for issuing, storing, discovery, and validating certificates in networks are presented in [3], [4], [5], [6], [7], [8], [9], [10], [11]. In a large scale network such as today's Internet, one cannot expect to have a central authority to issue, store, and validate all the certificates. A distributed system, where each user participates in issuing, storing, and validating certificates is desirable in such a network.

In [12] and [13], distributed architectures for issuing certificates, particularly in mobile networks, are presented.

In [12], Zhou and Haas present an architecture for issuing certificates in an ad-hoc network. According to this architecture, the network has $k$ servers. Each server has a 
different share of some private key $r k$. To generate a certificate, each server uses its own share of $r k$ to sign the certificate. If no more than $t$ servers have suffered from Byzantine failures, where $k \geq 3 t+1$, then the resulting certificate is correctly signed using the private key $r k$, thanks to threshold cryptography. The resulting certificate can be verified using the corresponding public key, which is known to every node in the ad hoc network.

In [13], Kong et al. presented another distributed architecture for issuing certificates. Instead of employing $k$ servers in the ad hoc network, no special nodes such as servers are in the network and every node in the network is provided with a different share of the private key $r k$. For a node $u$ to issue a certificate, the node $u$ forwards the certificate to its neighbors and each of them sign the certificate using its share of $r k$. If node $u$ has at least $t+1$ correct neighbors (i.e., they have not suffered from any failures), then the resulting certificate is correctly signed using the private key $r k$.

Both works assume that a certificate is signed by a special private key of an authority, and distribute the private key among many servers or nodes. By contrast, in [14] and this paper, we propose a distributed architecture where every node has both a public key and a private key so it can issue certificates for any other node in the network. This architecture is very efficient in issuing and validating certificates but cannot tolerate Byzantine failures. In particular, if one node suffers from Byzantine failure, then this node can successfully impersonate any other node that is reachable from this node in the certificate graph of the network. This vulnerability to Byzantine failures is not unique to our certificate work. In fact, many proposed certificate architectures, e.g., [3], [4], while [13], [5], [11], [10] yield similar vulnerabilities. Recently, we have identified a metric to evaluate the damage from this type of attacks. We call it "vulnerability" of the certificate system and discuss it in more details in [15].

In [11], Li et al. presented a role-based trust management language $R T_{0}$ and suggested the use of strongly typed distributed certificate storage to solve the problem of certificate chain discovery in distributed storage. However, they do not discuss how to efficiently assign certificates among the distributed storages. By contrast, our work focuses on minimizing storage overhead in certificate dispersal among the users while they have enough certificates so that there is no need for certificate chain discovery.

In [16], Ajmani et al. presented a distributed certificate storage using a peer-to-peer distributed hash table. This work assumes dedicated servers host an SDSI certificate directory and focuses on fast lookup service and load balancing among the servers. By contrast, our work assigns certificates to users such that there is no need for lookup and there are no dedicated certificate storage servers. Our work also focuses on efficient use of storages in all users in network.

In [17], Reiter and Stubblebine investigated how to increase assurance on authentication with multiple independent certificate chains. They introduce two types of independent chains, disjoint paths (no edge is shared by any two chains) and k-connective paths ( $\mathrm{k}$ certificates need to be compromised to disconnect all these paths). This paper shows that there are no polynomial-time algorithms for locating maximum sets of paths with these properties and presents approximation algorithms.

Perhaps the closest work to ours is [18], in which Hubaux et al. investigated how to disperse certificates in a certificate graph among the network nodes under two conditions. First, each node stores the same number of certificates. Second, with high probability, if two nodes meet, then they have enough certificates for each of them to obtain the public key of the other. By contrast, our work in [14] and here are based on two different conditions. First, different nodes may store different number of certificates, but the average number of certificates stored in nodes is minimized. Second, it is guaranteed (i.e., with probability 1 ) that, if two nodes meet, then they have enough certificates for each of them to obtain the public key of the other (if there exists a chain between them in the chain set).

Later, the same authors showed in [19] that a lower bound on the number of certificates to be stored in a node is $\sqrt{n}-1$, where $n$ is the number of nodes in the system. By contrast, we showed in [14] that the tight lower bound on the average number of certificates to be stored in a node is $e / n$, where $e$ is the number of certificates in the system. Our work here shows that finding an optimal dispersal of a given chain set is NP-complete and presents three polynomial-time algorithms which compute optimal dispersal of chain sets in three classes of practical interests and two extensions of these algorithms for more general classes of chain sets.

Zheng et al. presented algorithms that compute optimal dispersals for strongly connected graphs and directed graphs in [20]. The same authors also showed the tight upper bounds in these two classes of certificate graphs.

\section{Conclusion}

We have shown that, in general, finding an optimal dispersal of a given chain set is NP-complete. We have also discussed three polynomial-time algorithms, each of which computes an optimal dispersal for a rich class of chain sets and two extensions of these algorithms for two more general classes of chain sets. In [21], we have presented more polynomial-time algorithms, which compute an optimal dispersal for more classes of chain sets. This result can be used in any network setting. However, these algorithms are particularly useful when the network is large. In a large scale network such as today's Internet, one cannot expect to have a central authority for storing and distributing certificates among all users in the network. Instead, users can store a subset of certificates in the network so that any user can obtain the public key of the other whom the user wants to securely communicate with (if there was a chain in the chain set of the network). Moreover, in a large scale network, not all certificate chains in a certificate graph are in use. Computing an optimal dispersal of a chain set instead of the chain set derivable from the certificate graph of the network reduces the cost of dispersal.

This result can be also used as a metric to evaluate certificate graphs. The optimal dispersal cost is an important property of a certificate graph, since it affects the 
storage requirement of each node in the network. This is especially important in ad-hoc networks, where mobile nodes may be more restricted in terms of storage than stable nodes can be.

\section{ACKNOWLEDGMENTS}

The authors would like to thank Dr. Mustaque Ahamad, Dr. Xiaodong Zhang, and the anonymous reviewers for their valuable comments, as well as Jean-Philippe Martin for interesting discussions on the NP-completeness proof. This material is based upon work supported by the US National Science Foundation under Grant No. 0520250. Research of the second author is supported by NSERC Canada under grant number OGP 36899.

\section{REFERENCES}

[1] M.G. Gouda and E.E. Jung, "Stabilizing Certificate Dispersal," Proc. Symp. Self Stabilizing Systems, 2005.

[2] E. Jung, E.S. Elmallah, and M.G. Gouda, "Optimal Dispersal of Certificate Chains," Technical Report TR06-14, Dept. of Computer Sciences, Univ. of Texas at Austin, 2006.

[3] R.L. Rivest and B. Lampson, "SDSI-A Simple Distributed Security Infrastructure," Proc. Int'l Cryptology Conf. (Crypto '96) Rump Session, 1996.

[4] S. Boeyen, T. Howes, and P. Richard, "Internet X.509 Public Key Infrastructure Operational Protocols-LDAPv2," RFC 2559, 1999.

[5] M. Myers, R. Ankney, A. Malpani, S. Galperin, and C. Adams, "X.509 Internet Public Key Infrastructure Online Certificate Status Protocol-OCSP," RFC 2560, 1999.

[6] C. Ellison, B. Frantz, B. Lampson, R. Rivest, B. Thomas, and T. Ylonen, "SPKI Certificate Theory," RFC 2693, 1999.

[7] M. Blaze, J. Feigenbaum, J. Ioannidis, and A. Keromytis, "The KeyNote Trust-Management System Version 2," RFC 2704, 1999.

[8] D. Clarke, J.-E. Elien, C. Ellison, M. Fredette, A. Morcos, and R. Rivest, "Certificate Chain Discovery in SPKI/SDSI," J. Computer Security, vol. 9, no. 4, pp. 285-322, 2001.

[9] Y. Elley, A. Anderson, S. Hanna, S. Mullan, R. Perlman, and S. Proctor, "Building Certificate Paths: Forward vs. Reverse," Proc. Network and Distributed System Security Symp. (NDSS '01), pp. 153160, Feb. 2001

[10] E. Freudenthal, T. Pesin, L. Port, E. Keenan, and V. Karamcheti, "dRBAC: Distributed Role-Based Access Control for Dynamic Coalition Environments," Proc. 22nd Int'l Conf. Distributed Computing Systems (ICDCS '02), pp. 411-420, 2002.

[11] N. Li, W.H. Winsborough, and J.C. Mitchell, "Distributed Credential Chain Discovery in Trust Management," J. Computer Security, vol. 11, no. 1, pp. 35-86, 2003.

[12] L. Zhou and Z.J. Haas, "Securing Ad Hoc Networks," IEEE Network, vol. 13, no. 6, pp. 24-30, Nov.-Dec. 1999

[13] J. Kong, P. Zerfos, H. Luo, S. Lu, and L. Zhang, "Providing Robust and Ubiquitous Security Support for Wireless Mobile Networks," Proc. Ninth Int'l Conf. Network Protocols (ICNP '01), pp. 251-260, 2001.

[14] M.G. Gouda and E. Jung, "Certificate Dispersal in Ad-Hoc Networks," Proc. 24th Int'l Conf. Distributed Computing Systems (ICDCS '04), 2004.

[15] M.G. Gouda and E. Jung, "Vulnerability Analysis of Certificate Graphs," Int'l J. Security and Networks, vol. 1, no. 1, 2006.

[16] S. Ajmani, D.E. Clarke, C.-H. Moh, and S. Richman, "ConChord: Cooperative SDSI Certificate Storage and Name Resolution," Proc. Peer-to-Peer Systems: First Int'l Workshop (IPTPS '02), pp. 141-154, Mar. 2002.

[17] M.K. Reiter and S.G. Stubblebine, "Resilient Authentication Using Path Independence," IEEE Trans. Computers, vol. 47, no. 12, pp. 1351-1362, Dec. 1998.

[18] J.-P. Hubaux, L. Buttyán, and S. Capkun, "The Quest for Security in Mobile Ad Hoc Networks," Proc. 2001 ACM Int'l Symp. Mobile Ad Hoc Networking and Computing, pp. 146-155, 2001.

[19] S. Capkun, L. Buttyán, and J.-P. Hubaux, "Self-Organized PublicKey Management for Mobile Ad Hoc Networks," IEEE Trans. Mobile Computing, vol. 2, no. 1, pp. 52-64, Jan.-Mar. 2003.
[20] H. Zheng, S. Omura, J. Uchida, and K. Wada, "An Optimal Certificate Dispersal Algorithm for Mobile Ad Hoc Networks," Proc. Third Int'l Symp. Parallel and Distributed Computing/Third Int'l Workshop Algorithms, Models, and Tools for Parallel Computing on Heterogeneous Networks (ISPDC/HeteroPar '04), 2004.

[21] E. Jung, E.S. Elmallah, and M.G. Gouda, "Optimal Dispersal of Special Certificate Graphs," Proc. IEEE Global Telecomm. Conf. (Globecom '04), 2004.

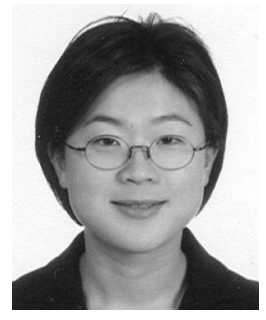

Eunjin (EJ) Jung graduated from Seoul National University in 1999 and received the MS and $\mathrm{PhD}$ degrees in computer sciences from the University of Texas at Austin in 2002 and 2006, respectively. She is currently an assistant professor at the Department of Computer Science at the University of lowa. Her research interests are security and fault-tolerance in computer networks and distributed systems. She is the winner of the 2006 William S. Livingston Outstanding Graduate Student Employee Award from the University of Texas at Austin. She is a member of the IEEE.

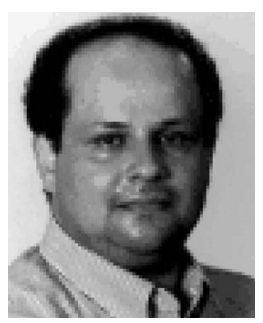

Ehab S. Elmallah received the BSc degree (1978) in computer science and automatic control from the University of Alexandria, the MSc degree (1984) in computing science from the University of Saskatchewan, and the $\mathrm{PhD}$ degree (1987) in computer science from the University of Waterloo. He is currently an associate professor in the Department of Computing Science at the University of Alberta. His research interests lie in the application of combinatorial and probabilistic methods in network design, with particular emphasis on resource management problems and security in wireless cellular networks, sensor networks, ad hoc networks, and optical networks. His research is supported by grants from NSERC, CITR, and CITO. He served on the technical program committee of numerous IEEE symposiums and workshops on communications and computer networks. He is a member of the IEEE and the Institute of Combinatorics and its Applications.

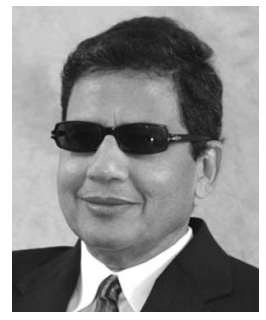

Mohamed G. Gouda received the BSc degrees in engineering and mathematics, both from Cairo University. Later, he received the MA degree in mathematics from York University and the master's and PhD degrees in computer science from the University of Waterloo. He worked for the Honeywell Corporate Technology Center in Minneapolis from 1977 to 1980 . In 1980, he joined the University of Texas at Austin, where he currently holds the Mike A. Myers Centennial Professorship in Computer Sciences. His research areas are distributed and concurrent computing and network protocols. In these areas, he has been working on abstraction, formality, correctness, nondeterminism, atomicity, reliability, security, convergence, and stabilization. He has published more than 60 journal papers and more than 80 conference and workshop papers. Professor Gouda won the 2001 IEEE Communications Society William R. Bennet Best Paper Award for his paper "Secure Group Communications Using Key Graphs," coauthored with C.K. Wong and S.S. Lam and published in the February 2000 issue of the IEEE/ACM Transactions on Networking (volume 8, number 1, pages 16-30). In 2004, his paper "Diverse Firewall Design," coauthored with Alex X. Liu and published in the Proceedings of the International Conference on Dependable Systems and Networks, won the William C. Carter Award. He is a member of the IEEE. More detailed information about the career of Professor Gouda can be found on his Web site, http://www.cs.utexas.edu/users/gouda.

$\triangleright$ For more information on this or any other computing topic, please visit our Digital Library at www.computer.org/publications/dlib. 